\title{
Systematic review of literature and analysis of big data from the National Health Insurance System on primary immunodeficiencies in Korea
}

\author{
Sohee Son, $\mathrm{PhD}^{1}$, Ji-Man Kang, MD, PhD², Younsoo Hahn, MD, PhD³ , Kangmo Ahn, MD, PhD¹, Yae-Jean Kim, MD, PhD ${ }^{1}$ \\ ${ }^{1}$ Department of Pediatrics, Samsung Medical Center, Sungkyunkwan University School of Medicine, Seoul, Korea; ${ }^{2}$ Department of Pediatrics, Severance Children's \\ Hospital, Yonsei University College of Medicine, Seoul, Korea; ${ }^{3}$ Department of Pediatrics, Chungbuk National University Hospital, Cheongju, Korea
}

There are very scant data on the epidemiology of primary immunodeficiency diseases (PIDs) in Korea. Here we attempted to estimate the PID epidemiology and disease burden in Korea. A systematic review was performed of studies retrieved from the PubMed, KoreaMed, and Google Scholar databases. Studies on PIDs published in Korean or English between January 2001 and November 2018 were analyzed. The number of PID patients and the healthcare costs were estimated from Health Insurance Review and Assessment Service (HIRA) Korea data for 2017. A total of 398 PID patients were identified from 101 reports. Immunodeficiencies affecting cellular and humoral immunity were reported in 11 patients, combined immunodeficiency with associated or syndromic features in 40 , predominantly antibody deficiencies in 144, diseases of immune dysregulation in 58 , congenital defects of phagocytes in 104 , defects in the intrinsic and innate immunity in 1 , auto-inflammatory disorders in 4, complement deficiencies in 36 , and phenocopies of PID in none. From the HIRA reimbursement data, a total of 1,162 outpatients and 306 inpatients were treated for 8,166 and 6,149 days, respectively. In addition, reimbursement was requested for 8,200 outpatient and 1,090 inpatient cases and \$1,924,000 and $\$ 4,715,000$ were reimbursed in 2017 , respectively. This study systematically reviewed published studies on PID and analyzed the national open data system of the HIRA to estimate the disease burden of PID, for the first time in Korea.

Key words: Primary immunodeficiency diseases, Epidemiology

\section{Key message}

In this article, a systematic review of reported primary immunodeficiency disease (PID) cases in Korea was performed and we attempted to estimate the number of PID patients and healthcare costs for the first time in Korea. Our review revealed that Korean PID cases are greatly underreported in the literature based on health insurance. Physicians in the field and health care policymakers should be aware of the disease burden of PID.

\section{Introduction}

Primary immunodeficiency diseases (PIDs) are inborn errors of immunity caused by a single-gene mutation. Almost 300 PIDs have been identified to date, and more continue to be discovered. Patients with PID are known to have increased susceptibility to infection caused by usual or unusual pathogens. However, phenotypes of PID are diverse with additional features such as malignancy, allergy, autoimmunity, and autoinflammation.

\section{Global and national epidemiology of PIDs}

PIDs are considered rare diseases. However, with recent advances in diagnostics, increased awareness, improved care quality, and registry projects in many countries and continents, the number of identified PID patients is increasing. There are several well-established PID patient registries in America, Africa, and Europe (Table 1). ${ }^{1-3)}$ There is a movement for the development of a PID registry in the Asia-Pacific region, and Japan already has a well-established PID registry (Table 1).4)

Growing evidence suggests that PID might be more common than generally thought. A study from 2013 estimated that, although there are as many as 6 million people with PID, less than $1 \%$ have been identified. ${ }^{1)}$ There have been many attempts to estimate the epidemiology of PID in many countries with wide variability. For example, the prevalence of PID was estimated at 5.6/100,000 in Australia and New Zealand in 2007,5) 5.38/ $100,000$ inhabitants in France in 2011, 6,7$)$ and 1.94/100,000 in the United Kingdom in 2011.6,7) These estimates of prevalence may be lower than the actuals because these numbers were drawn from registries.

Globally, the Jeffrey Modell Foundation has established a network of physician experts on PID (Jeffrey Modell Centers Network) and performs an annual global survey. From the last survey report in 2014, as of 2013, 138,847 patients were being followed by PID expert physicians and 77,192 patients were

Corresponding author: Yae-Jean Kim, MD, PhD. Department of Pediatrics, Samsung Medical Center, Sungkyunkwan University, School of Medicine, Seoul 06351, Korea 凶Email: yaejeankim@skku.edu, https://orcid.org/0000-0002-8367-3424

Received: 18 October, 2019, Revised: 1 June, 2020, Accepted: 3 June, 2020

This is an open-access article distributed under the terms of the Creative Commons Attribution Non-Commercial License (http://creativecommons.org/licenses/bync/4.0/) which permits unrestricted non-commercial use, distribution, and reproduction in any medium, provided the original work is properly cited.

Copyright (c) 2021 by The Korean Pediatric Society 
confirmed as having PID in surveys collected from 225 centers in 78 countries in 6 continents. ${ }^{6}$ In 2013 , there were $74.1 \%$ and $27.9 \%$ increases in the number of patients followed and those with an identified PID, respectively, compared to 2011. ${ }^{6}$

In 2017, the physicians reported a total of 187,988 patients with PID, with an estimated global prevalence of 2.5 per 100,000 persons. ${ }^{8,9)}$

In Japan, a survey was conducted on PID patients who were alive on December 1,2008 and those who were newly diagnosed and dead between December 1, 2007 and November 30, 2008. This study estimated the prevalence of PID as 2.3 per 100,000 persons (Table 1). ${ }^{4)}$

In Korea, a study investigated the epidemiology of PID from 2001 to 2005 and identified 152 patients from 23 major hospitals. ${ }^{10)}$ In this study, the prevalence of PID in Korea in 2005 was 1.13 per 100,000 persons. However, these numbers may have been underestimated; thus, further studies are needed.

\section{Advancement in diagnosis and management of PID}

Certain PIDs have typical presentations, but the clinical manifestations among many PIDs are variable, although they are also similar. Therefore, taking a diagnostic approach according to phenotypic classification helps clinicians greatly when faced with potential PID. ${ }^{11)}$ Samples from PID patients are often evaluated using flow cytometry analysis. In Korea, basic lymphocyte subset analysis (T, B, NK cells, and monocytes) by flow cytometry is relatively readily available in many referral centers, whereas tests such as dihydrorhodamine to screen for chronic granulomatous disease (CGD) are currently available only in 3 centers in the nation. In addition, several other functional tests are not available in the clinical laboratories of Korean hospitals. PID patients are in urgent need of a national insurance benefit for immunology work-ups. For example, the cost for lymphocyte subset analysis is not properly reimbursed, even for patients with severe combined immunodeficiency (SCID) or X-linked agammaglobulinemia (XLA), and deferred automatically in an electronic reimbursement system by the national health insurance system. However, it is reimbursed for cancer patients who undergo hematopoietic cell transplantation (HCT) and follow-up tests during the post-HCT period. Increased awareness is warranted among physicians in the clinical field and officers at the national health insurance review agency to increase the care benefit of PID patients at the diagnostic workup stage and during their disease follow-up period. Therefore, support and relevant health policies are needed at the government level.

Since PIDs are typical examples of single-gene inborn errors of immunity, the genetic diagnosis is crucial for the final confirmation of the disease. It is noteworthy that, according to Casanova et al., ${ }^{12)}$ up to 49 of the 232 monogenetic etiologies (21\%) of human PIDs were initially reported in single patients. With the advancement of genetic analysis technology in the next-generation sequencing era, the cumulative number of single-gene defects underlying PIDs has increased in a short period of time. ${ }^{13)}$ However, next-generation sequencing does not analyze all of the unknown causes of underlying genetic mutations, and 15\%-40\% of those unknown cases are solved. ${ }^{14)}$ Therefore, clinicians must detect clinical clues and characterize each phenotype.

Traditional management strategies of PID patients include immunoglobulin (Ig) replacement, HCT, and antimicrobial prophylaxis. Gene therapy has been also attempted in the field. ${ }^{15,16)}$ With advances in genetic diagnosis and basic immunology, there

Table 1. Estimated epidemiology of PID in Korea and other registries according to the 2017 IUIS Phenotypic Classification

\begin{tabular}{|c|c|c|c|c|c|}
\hline Varible & $\begin{array}{c}\text { Systematic } \\
\text { review }^{\text {a) }}\end{array}$ & HIRA data ${ }^{\text {b) }}$ & $U S^{c)}$ & $E U^{d)}$ & $J^{\prime a p a n}{ }^{e)}$ \\
\hline Total (estimates) & 398 & 1,162 & 5,346 & 19,355 & 2,900 \\
\hline General population, 2019 & & $51,833,175$ & $329,676,200$ & $512,600,000$ & $125,941,289$ \\
\hline Prevalence (per 100,000) & & 2.2 & 1.6 & 3.8 & 2.3 \\
\hline I. Immunodeficiencies affecting cellular and humoral immunity (SCID, CID, etc.) & $11(3)$ & $42(4)$ & $513(10)$ & $1,445(7)$ & $93(7)$ \\
\hline II. CID with associated or syndromic features (WAS, DiGeorge, HIES, etc.) & $37(10)$ & $43(4)$ & $1,076(20)$ & $2,693(14)$ & 194(16) \\
\hline III. Predominantly antibody deficiencies (XLA, etc.) & $143(36)$ & $460(40)$ & $2,663(50)$ & $10,966(57)$ & $501(40)$ \\
\hline IV. Diseases of immune dysregulation (HLH, CTLA-4, etc.) & $58(15)$ & NA & $225(4)$ & $753(4)$ & $49(4)$ \\
\hline V. Congenital defects of phagocyte (CGD, etc.) & $104(26)$ & $124(11)$ & $638(12)$ & $1,689(9)$ & $230(19)$ \\
\hline VI. Defects in Intrinsic and Innate immunity & $1(0.3)$ & \multirow{4}{*}{$\begin{array}{l}\text { PID with other } \\
\text { defects, } 233 \\
\text { (20); Others, } \\
260 \text { (11) }\end{array}$} & $95(2)$ & $193(1)$ & $15(1)$ \\
\hline VII. Auto-inflammatory disorders (FMF, etc.) & $4(1)$ & & NA & $398(2)$ & $108(9)$ \\
\hline VIII. Complement deficiencies (aHUS, etc.) & $36(9)$ & & $26(0.5)$ & $946(5)$ & $32(3)$ \\
\hline IX. Phenocopies of PID & $0(0)$ & & Others: 110 & $272(1)$ & $18(1)$ \\
\hline
\end{tabular}

Values are presented as number or number (\%).

PID, primary immunodeficiency disease; IUIS, International Union of Immunological Societies; SCID, severe combined immunodeficiency; CID, combined immunodeficiency; WAS, Wiskott Aldrich syndrome; HIES, hyper immunoglobulin E syndrome; XLA, X-linked agammaglobulinemia; HLH, hemophagocytic lymphohistiocytosis; CTLA-4, hyper immunoglobulin E syndrome; CGD, chronic granulomatous disease; FMF, familial mediterranean fever; aHUS, atypical hemolytic uremic syndrome; NA, not available.

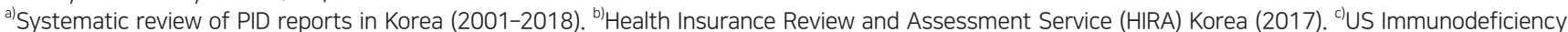


diseases in Japan (2007-2008). 
are opportunities for newer therapeutic options using targeted therapy at the molecular level. In 2018, the Nobel Prize was given to one of the researchers who worked on the CTLA-4 gene. ${ }^{17)}$ Although much attention was given to the application of CTLA-4 for cancer treatment, PID patients with the CTLA-4 mutation can also benefit from target therapy using CTLA-4 Ig treatment. ${ }^{18,19)}$ The current Korean health insurance system was mainly designed to enable the reimbursement for common diseases with high numbers of patients such as diabetes, hypertension, or cancers. In more common diseases such as cancers, many advanced target therapies at the molecular level are being used in the clinical field in Korea. However, there are many hurdles for rare PID patients (e.g., only one patient diagnosed in Korea) who may need this type of special target therapy as well as reimbursement from the Korean government system. There should be a formal and easy to approach channel in Health Insurance Review and Assessment Service (HIRA) with staff designated to manage rare diseases issues to ensure efficient communication and eventually benefits for patients with rare diseases who also have the right to receive the best care possible in Korea. Therefore, there is a need to estimate the disease burden of PID in Korea and identify advanced treatment options in Korean PID patients. This is also achievable at the global level given the fact of the rarity of the diseases and PID experts constantly collaborate to provide the world cohort data in the literature ${ }^{20)}$; Korean patient data should be analyzed internationally to provide evidence for a therapeutic approach in Korea.

The number of physicians with advanced knowledge about PID and proper training for managing these special groups of diseases is very limited in Korea; thus, many PID patients do not obtain a timely accurate diagnosis. Constant efforts including public awareness, physician education, and cross-talk with a government agency should be made to advance PID care in Korea.

The National Institutes of Health (NIH) is the largest public funder of biomedical research worldwide. ${ }^{21)} \mathrm{NIH}$ grants for PID were provided for 409 projects $(\$ 35,410,126)$ in the United States (US) in 2016 and 2017.22) Among them, 2 NIH grants $(\$ 697,747)$ were provided for the US Immunodeficiency Network (USIDNET) in 2016 and 2017. ${ }^{23)}$ The USIDNET was established to advance scientific research on PID in the US. ${ }^{24)}$ One of their main areas of focus is to assemble and maintain a registry of validated data from PID patients. The USIDNET has been continuously funded from the National Institute of Allergy and Infectious Diseases of the NIH since 2005. In Korea, one such project aiming to estimate the prevalence of PID was supported by a national grant from the Korea Centers for Disease Control \& Prevention (KCDC), which reported the results in 2006. ${ }^{25)}$ However, there is no continuous ongoing PID registry funded by a national grant in Korea.

\section{Systematic review of PID reports in Korea}

All Korean or English studies on PIDs reported in a Korean or international scientific journal between January 2001 and November 2018 were identified. The International Union of Immunological Societies (IUIS) PID expert committee developed the phenotypic classification to help clinicians diagnose PID at the bedside. This report represents the most current and complete catalog of known PIDs. ${ }^{11)}$ Thus, it serves as a reference for these conditions and provides a framework to help in the

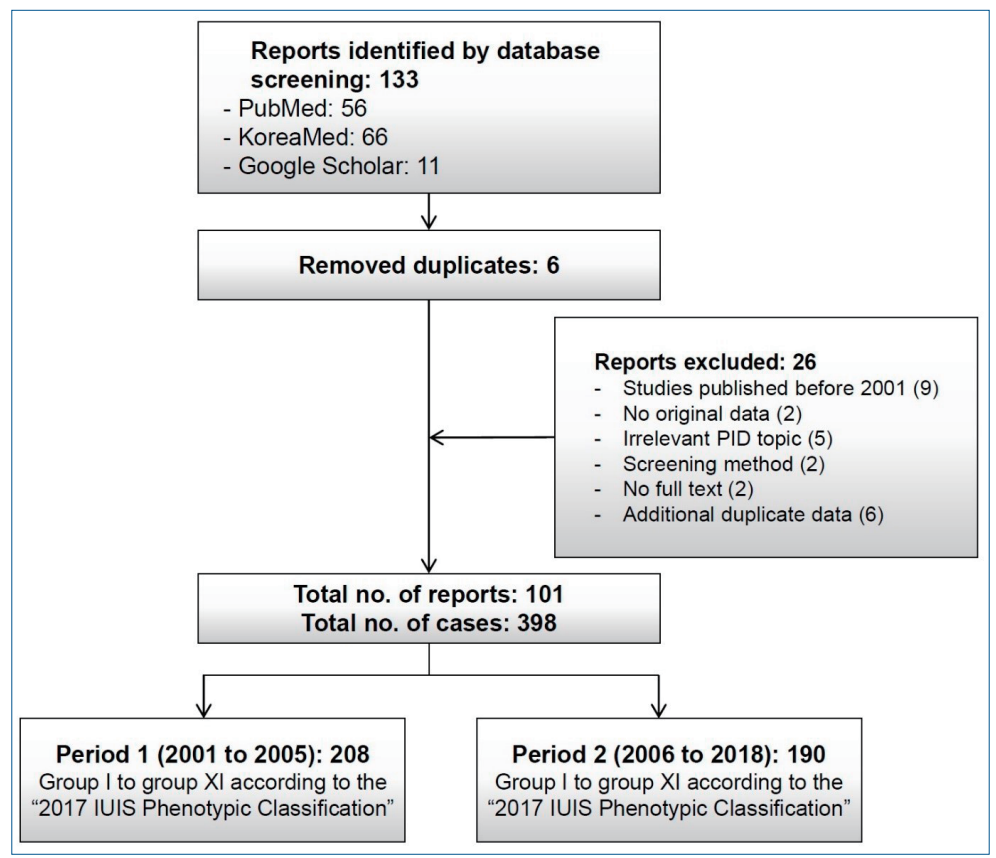

Fig. 1. Flowchart of the article selection process for this systematic review of primary immunodeficiencies in Korea. PID, primary immunodeficiency disease; IUIS, International Union of Immunological Societies. 
diagnostic approach to patients with suspected PID in a publication every 2 years. We searched the PubMed, KoreaMed, and Google Scholar databases for systematic reviews and checked for duplicates (Fig. 1). The diagnosis of PID was categorized from group I to group XI according to the 2017 IUIS Phenotypic Classification. The diagnosis of PID was divided into period 1, 2001-2005, and period 2, 2006-2018, because a multicenter study estimated PID epidemiology from 2001 to 2005 in Korea.

A total of 398 PID patients were identified in 101 reports. Of them, 208 (27 reports) and 190 (74 reports) were in periods 1 and 2, respectively (Fig. 1; Tables 1, 2). The most frequent PIDs were as follows: V. congenital defects of phagocyte b: functional defects $(n=94,23.6 \%)$, III. predominantly antibody deficiencies a: hypogammaglobulinemia ( $n=82,20.6 \%)$, III. predominantly antibody deficiencies $\mathrm{b}$ : other antibody defici- encies ( $\mathrm{n}=62,15.6 \%)$, and IV. disease of immune dysregulation a: hemophagocytic lymphohistiocytosis (HLH) \& Epstein-Barr virus susceptibility $(\mathrm{n}=53,13.3 \%)$. Among the diseases, CGD was the most frequently reported PID $(n=86,21.6 \%)$ (data not shown). No cases were reported of patients with VI. Defects in intrinsic and innate immunity b: Mendelian susceptibility to mycobacterial disease and viral infection, VIIb. auto-inflammatory disorders, and IX. phenocopies of PID.

Among the 10 SCID cases, 5 (50.0\%) were reported at Samsung Medical Center (data not shown). ${ }^{26,27)}$ Among the 53 cases of HLH, 20 (37.7\%) were reported from Asan Medical Center. Among the 86 CGD cases, 46 (53.5\%) were reported from Seoul National University. ${ }^{10,28,29)}$ CTLA4 deficiency $(\mathrm{n}=5$, $1.0 \%)$ and familial Mediterranean fever $(\mathrm{n}=4,1.0 \%)$ were not found in period 1 but were reported in period 2 .

Table 2. Number of cases of primary immunodeficiencies in Korea in the literature

\begin{tabular}{|c|c|c|c|c|}
\hline IUIS classification & Primary immunodeficiencies & $\begin{array}{c}\text { Period } 1 \\
(2001-2005)\end{array}$ & $\begin{array}{c}\text { Period 2 } \\
(2006-2018)\end{array}$ & $\begin{array}{l}\text { No. of } \\
\text { cases }\end{array}$ \\
\hline \multicolumn{5}{|l|}{ I. Immunodeficiencies affecting cellular and humoral immunity } \\
\hline (A) Severe combined immunodeficiencies (SCID) & SCID & 7 & 3 & 10 \\
\hline (B) Combined Immunodeficiencies & NA & 1 & 0 & 1 \\
\hline \multicolumn{5}{|l|}{ II. CID with associated or syndromic features } \\
\hline $\begin{array}{l}\text { (A) Congenital thrombocytopenia, immunoosseous dysplasia, } \\
\text { thymic defects with additional congenital anomalies }\end{array}$ & Wiskott Aldrich Sd, DiGeorge Sd, CHARGE Sd & 8 & 21 & 29 \\
\hline $\begin{array}{l}\text { (B) Hyper-IgE Sd (HIES), dyskeratosis congenita, defects of } \\
\text { vitamin B12 and folate metabolism, anhidrotic ectoder- } \\
\text { modysplasia with immunodeficiency, others }\end{array}$ & HIES, Comel Neterton Sd & 5 & 4 & 11 \\
\hline \multicolumn{5}{|l|}{ III. Predominantly antibody deficiencies } \\
\hline (A) Hypogammaglobulinemia & $\begin{array}{l}\text { X-linked agammaglobullinemia (BTK), CVID, acti- } \\
\text { vated phosphoinositide } 3 \text { kinase delta Sd (APDS) }\end{array}$ & 56 & 26 & 82 \\
\hline (B) Other antibody deficiencies & $\begin{array}{l}\text { Selective IgA deficiency, IgG subclass deficiency } \\
\text { with IgA deficiency, Isolated IgG subclass defici- } \\
\text { ency, Hyper IgM Sd }\end{array}$ & 57 & 5 & 62 \\
\hline
\end{tabular}

IV. Diseases of immune dysregulation



VI. Defects in Intrinsic and Innate immunity

$\begin{array}{llrr}\text { (A) Bacterial and Parasitic Infections } & \text { NA } & 0 & 1 \\ \text { (B) MSMD and Viral infection } & \text { NA } & 0 & 0\end{array}$

VII. Auto-inflammatory disorders

(A) Recurrent inflammation, Systemic inflammation with urti- FMF carial rash, Others

(B) Sterile inflammation (skin/bone/joints), type 1 Interfero- NA nopathies

VIII. Complement deficiencies

Complement deficiencies

C1 C3 C4 C5 C7 C9 def. atypical hemolytic uremic Sd 13

$23-36$

IX. Phenocopies of PID

Phenocopies of PID

NA

Total

$\begin{array}{rrr}0 & 0 & 0 \\ 208 & 190 & 398\end{array}$

PID was categorized into groups I-XI according to the 2017 IUIS Phenotypic Classification.

PID, primary immunodeficiency disease; IUIS, International Union of Immunological Societies; NA, not available; CID, combined immunodeficiency; Sd, syndrome; CVID, common variable immunodeficiency; HLH, hemophagocytic lymphohistiocytosis; EBV, Epstein-Barr virus; CGD, chronic granulomatous disease; LAD I, leukocyte adhesion deficiency-1; MSMD, Mendelian susceptibility to mycobacterial disease; FMF, familial mediterranean fever. 
In VIII. complement deficiencies, 13 cases of C7 and C9 deficiency and atypical hemolytic uremic syndrome (aHUS) and 23 cases of C1, C3, C4, C5, C7, and C9 deficiency and aHUS were defined in periods 1 and 2, respectively.

\section{HIRA reimbursement data}

The number of PID patients, treatment days, and reimbursement requests as well as the healthcare costs were estimated for 1 year in 2017 using data on the HIRA website (http://opendata. hira.or.kr/).

A total of 1,162 outpatients and 306 inpatients were treated for 8,166 and 6,149 days, respectively; 124 outpatients (10.7\%) and 49 inpatients $(16.0 \%)$ were treated for functional defect of neutrophils (FDN), 460 (39.6\%) and 177 (57.8\%) for predominantly antibody deficiency (PAD), 42 (3.6\%) and 5 (1.6\%) for combined immunodeficiency (CID), 233 (20.1\%) and 28 (9.2\%) for immunodeficiency associated with other major defects (IDMD), 43 (3.7\%) and 18 (5.9\%) for common variable immunodeficiency (CVID), and 260 (22.4\%) and 29 (9.5\%) for other immunodeficiencies (Others), respectively (Fig. 2A, Table 1).

Treatment was delivered for 8,166 and 6,149 days for outpatients and inpatients, respectively: 1,249 (15.3\%) and 1,357 (22.1\%) for patients with FDN, 3,175 (38.8\%) and 672 (37.3\%) in patients with $\mathrm{PAD}, 225(2.7 \%)$ and $21(4.5 \%)$ in patients with CID, 2,188 (26.8\%) and 118 (21.1\%) in patients with IDMD, $496(6.0 \%)$ and 68 (7.5\%) in patients with CVID, and 855
(10.4\%) and 80 (7.5\%) in patients with Others, respectively (Fig. 2B).

Reimbursement was requested in 8,200 outpatient care episodes and 1,090 inpatient care episodes: 1,261 (15.4\%) and 131 (12.0\%) for FDN, 3,175 (38.7\%) and 672 (61.7\%) for PAD, 225 (2.7\%) and 21(1.9\%) for CID, 2,188 (26.7\%) and 118 (10.8\%) for IDMD, 496 (6.0\%) and 68 (6.2\%) for CVID, and $855(10.4 \%)$ and $80(7.3 \%)$ for Others for outpatient and inpatient care episodes, respectively (Fig. 2C).

Total healthcare cost (reimbursement plus patient copayment) was estimated as $\$ 1,024,000$ for outpatient care and $\$ 2,500,000$ for inpatient care in 2017: $\$ 188,000$ and $\$ 877,000$ for FDN, $\$ 502,000$ and $\$ 647,000$ for PAD, $\$ 39,000$ and $\$ 183,000$ for CID, $\$ 140,000$ and $\$ 466,000$ for IDMD, $\$ 105,000$ and $\$ 142,000$ for CVID, and $\$ 50,000$ and $\$ 186,000$ for Others, respectively (Fig. 2D).

In an outpatient care setting, patients with PAD $(n=460)$ had the highest number of treatment visit days and the highest number of reimbursement requests, and spent the largest amount of healthcare cost (Fig. 2). In an inpatient care setting, patients with PAD $(n=177)$ had the highest number of inpatient treatment days and highest number of reimbursement requests, while patients with FDN incurred the greatest healthcare costs.

The claims data of the HIRA is an important information source for healthcare service research in Korea. ${ }^{30)}$ Despite its importance, there are a few limitations: specifically, these data may involve insufficient cases for rare diseases such as PID and data for a certain age group may be at lower frequency.

In the HIRA database, Korean Standard Classification of

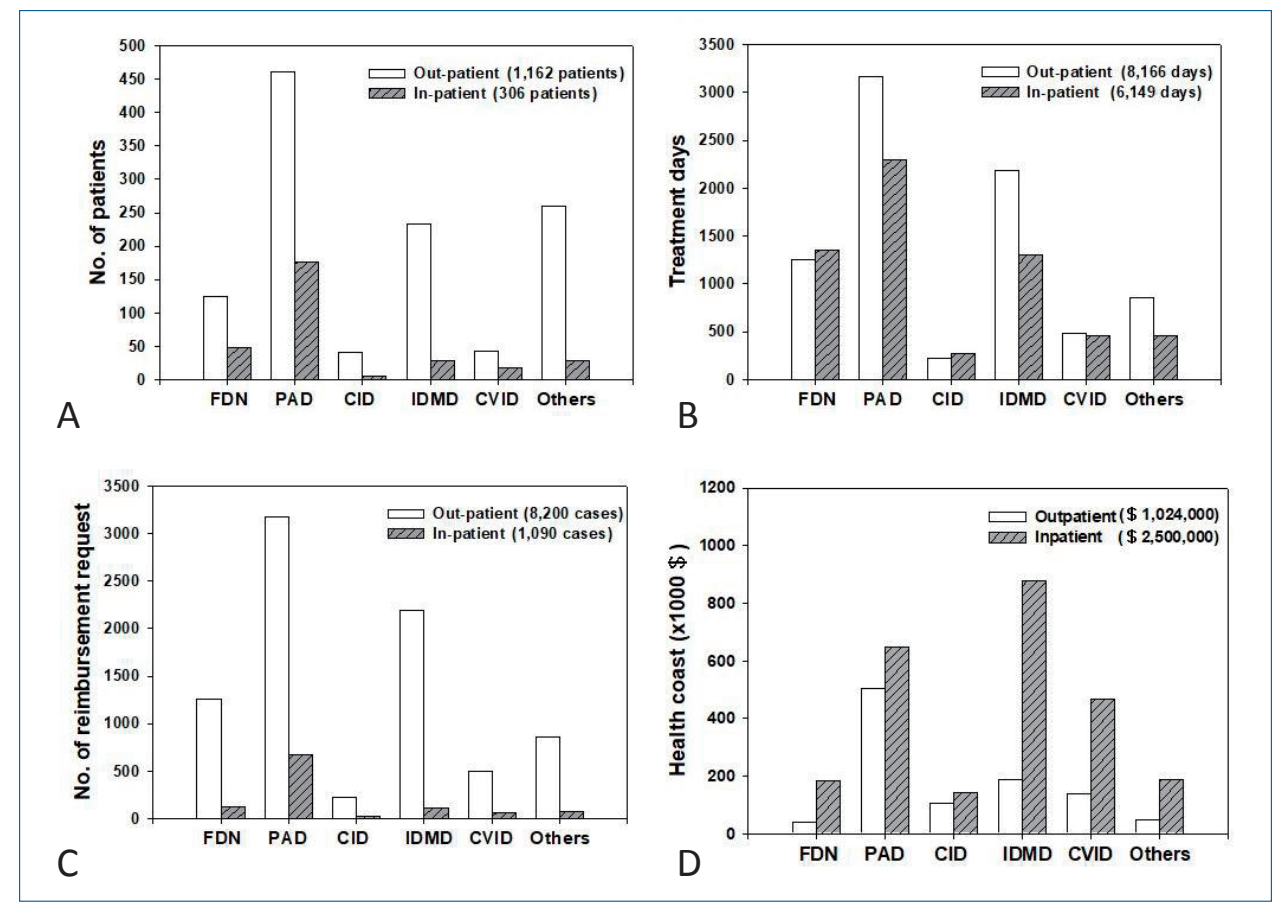

Fig. 2. Primary immunodeficiency disease (PID) patients who requested reimbursement in the HIRA Korea (2017). (A) Number of patients. (B) Number of treatment days. (C) Number of reimbursement requests. (D) Healthcare costs for PID. CID, combined immunodeficiency; CVID, common variable immunodeficiency; FDN, functional defect of neutrophils; Healthcare cost, reimbursement plus patient copayment; IDMD, immunodeficiency associated with other major defects; Others, other immunodeficiencies; PAD, predominantly antibody deficiency. 
Disease Version 7 (KCD-7) codes are used. This is the Korean translation of the International Statistical Classification, 10th Revision (ICD-10) produced by the World Health Organization (WHO). ${ }^{31,32)}$

In the HIRA database, statistics for the following 6 main KCD7 codes for PID are as follows: FDN (D71), PAD (D80), CID (D81), IDMD (D82), CVID (D83), and Others (D84). These main codes have several subcategories. For example, according to ICD-10 (WHO version; 2016), PAD (D80) includes D80.0, hereditary hypogammaglobulinemia; D80.1, nonfamilial hypogammaglobulinemia; D80.2, selective deficiency of IgA; D80.3, selective deficiency of IgG; D80.4, selective deficiency of IgM; D80.5, immunodeficiency with increased IgM; D80.6, antibody deficiency with near-normal immunoglobulins or with hyperimmunoglobulinemia; D80.7, transient hypogammaglobulinemia of infancy; D80.8, other immunodeficiencies with predominantly antibody defects; and D80.9, immunodeficiency with predominantly antibody defects, unspecified.

CID (D81) includes D81.0, SCID with reticular dysgenesis; D81.1, SCID with low T- and B-cell numbers; D81.2, SCID with low or normal B-cell numbers; D81.3, adenosine deaminase deficiency; D81.4, Nezelof syndrome; D81.5, purine nucleoside phosphorylase deficiency; D81.6, major histocompatibility complex class I deficiency; D81.7, major histocompatibility complex class II deficiency; D81.8, other combined immunodeficiencies; and D81.9, combined immunodeficiency, unspecified. The subcategories above are also found and searchable in HIRA database statistics.

CVID (D83) includes D83.0, CVID with predominant abnormalities of B-cell numbers and function; D83.1, CVID with predominant immunoregulatory T-cell disorders; D83.2, CVID with autoantibodies to B- or T-cells; D83.8, other CVID; and D83.9, common variable immunodeficiency, unspecified.

Since about $40 \%$ of PID patients had an antibody deficiency, we took a closer look at the number of patients with PAD (agammaglobulinemia/other antibody deficiency [D80 and D80.0D80.9 in KCD-7 codes] and CVID [D83 and D83.0-D83.9]), the representative diseases for intravenous Ig replacement therapy (Fig. 3). The highest number of PAD patients plus CVID patients was observed in the 1st decade (Fig. 3A). Among them, XLA patients (D80.0 in KCD-7 codes) and CVID patients (D83 and D83.0-D83.9 in KCD-7 codes) are shown in Fig. 3B. The highest number of these patients were aged 20-29 years ( 29 and 14 patients, respectively).

Although IUIS classification is updated every 2 years, it has limited ability to reflect the changes in real time for PID coding to enable reimbursement in clinical practice. Several new diagnoses are not properly coded in the reimbursement system in many countries. To address this issue, PID experts should make continuous efforts to both update IUIS classification and apply the IUIS classification to ICD codes in cooperation with government agencies. Education for physicians in the field should also be updated with new PID ICD codes.

\section{Limitations}

We searched published studies on PID in 101 peer-reviewed medical journals. Among them, 85 reports (84.2\%) were written in Korean and 71 (70.3\%) were case reports. This study could not estimate the prevalence or regional distribution of specific PIDs. To avoid duplicate reimbursements, the health insurance data for 1 year (2017) were used to estimate the PID disease healthcare cost burden. However, this was the first systematic review of all available Korean reports on PID and the first report on healthcare coast for PID patients in Asia.

\section{Conclusion}

This article described our systematic review of all reported PID cases in Korea (published in Korean or English) in which we attempted to estimate the number of PID patients and healthcare costs for the first time in Korea using open big data in the national health insurance reimbursement system. Our review revealed that Korean PID cases are greatly underreported in the



Fig. 3. Number of patients with primary immunodeficiency diseases needed to estimate the need for intravenous immunoglobulin treatment. (A) primary immunodeficiency diseases (agammaglobulinemia/other antibody deficiency plus CVID). (B) XLA and CVID. Numbers on top of bars and over lines are the number of patients. CVID, common variable immunodeficiency; XLA, X-linked agammaglobulinemia. 
literature, and based on health insurance data, there are more than 1,000 PID patients in Korea. In addition, there is a definite need for increased awareness and education for the proper use of KCD-7 (the translated version of the ICD-10) for existing or newly discovered PIDs in the reimbursement system. Physicians in the field and health care policymakers should be aware of the disease burden of PID. To better estimate the epidemiology of PID and its disease burden in Korea, a national registry project is warranted at the government level.

\section{Conflicts of interest}

No potential conflict of interest relevant to this article was reported.

\section{Acknowledgments}

This study was supported by the Basic Science Research Program through the National Research Foundation of Korea (NRF) funded by the Ministry of Education (NRF-2016R1A6 A3A11932463).

\section{References}

1. Bousfiha AA, Jeddane L, Ailal F, Benhsaien I, Mahlaoui N, Casanova JL, et al. Primary immunodeficiency diseases worldwide: more common than generally thought. J Clin Immunol 2013;33:1-7.

2. The United States Immunodeficiency Network (USIDNET) [Internet]. Towson (MD): USIDNET; [cited 2019 June 27]. Available from: www. USIDNET.org.

3. Registry Working Party ESID Registry [Internet]. Amsterdam (The Netherlands): European Society for Immunodeficiencies (ESID); [cited 2019 Oct 7]. Available from: https://esid.org/Working-Parties/RegistryWorking-Party/ESID-Registry.

4. Ishimura M, Takada H, Doi T, Imai K, Sasahara Y, Kanegane H, et al. Nationwide survey of patients with primary immunodeficiency diseases in Japan. J Clin Immunol 2011;31:968-76.

5. Kirkpatrick P, Riminton S. Primary immunodeficiency diseases in Australia and New Zealand. J Clin Immunol 2007;27:517-24.

6. Modell V, Knaus M, Modell F, Roifman C, Orange J, Notarangelo LD. Global overview of primary immunodeficiencies: a report from Jeffrey Modell Centers worldwide focused on diagnosis, treatment, and discovery. Immunol Res 2014;60:132-44.

7. Espinosa-Rosales FJ, Condino-Neto A, Franco JL, Sorensen RU. Into action: improving access to optimum care for all primary immunodeficiency patients. J Clin Immunol 2016;36:415-7.

8. Modell V, Orange JS, Quinn J, Modell F. Global report on primary immunodeficiencies: 2018 update from the Jeffrey Modell Centers Network on disease classification, regional trends, treatment modalities, and physician-reported outcomes. Immunol Res 2018;66:367-80.

9. World Population Prospects: United Nations Department of Economic and Social Affairs (UN DESA). New York: United Nations; [cited 2017 June 21]. Available from: https://www.un.org/development/desa/publications/world-population-prospects-the-2017-revision.html.

10. Rhim JW, Kim KH, Kim DS, Kim BS, Kim JS, Kim CH, et al. Prevalence of primary immunodeficiency in Korea. J Korean Med Sci 2012;27:788-93.

11. Bousfiha A, Jeddane L, Picard C, Ailal F, Bobby Gaspar H, Al-Herz W, et al. The 2017 IUIS phenotypic classification for primary immunodeficiencies. J Clin Immunol 2018;38:129-43.

12. Casanova JL, Conley ME, Seligman SJ, Abel L, Notarangelo LD. Guidelines for genetic studies in single patients: lessons from primary immunodeficiencies. J Exp Med 2014;211:2137-49.
13. Meyts I, Bosch B, Bolze A, Boisson B, Itan Y, Belkadi A, et al. Exome and genome sequencing for inborn errors of immunity. J Allergy Clin Immunol 2016;138:957-69.

14. Al-Mousa H, Abouelhoda M, Monies DM, Al-Tassan N, Al-Ghonaium A, $\mathrm{Al}-\mathrm{Saud} \mathrm{B}$, et al. Unbiased targeted next-generation sequencing molecular approach for primary immunodeficiency diseases. J Allergy Clin Immunol 2016;137:1780-7.

15. Mamcarz E, Zhou S, Lockey T, Abdelsamed H, Cross SJ, Kang G, et al. Lentiviral gene therapy combined with low-dose busulfan in infants with SCID-X1. NEngl J Med 2019;380:1525-34.

16. Fischer A, Hacein-Bey Abina S, Touzot F, Cavazzana M. Gene therapy for primary immunodeficiencies. Clin Genet 2015;88:507-15.

17. Press release: the nobel prize in physiology or medicine 2018, a new principle for immune therapy: The Nobel Foundation [Internet]. Stockholm (Sweden): The Nobel Foundation; c2020 [cited 2018 Oct 1]. Available from: https://www.nobelprize.org/prizes/medicine/2018/pressrelease/.

18. Lee S, Moon JS, Lee CR, Kim HE, Baek SM, Hwang S, et al. Abatacept alleviates severe autoimmune symptoms in a patient carrying a de novo variant in CTLA-4. J Allergy Clin Immunol 2016;137:327-30.

19. Lo B, Zhang K, Lu W, Zheng L, Zhang Q, Kanellopoulou C, et al. Autoimmune disease. Patients with LRBA deficiency show CTLA4 loss and immune dysregulation responsive to abatacept therapy. Science 2015;349:436-40.

20. Egg D, Schwab C, Gabrysch A, Arkwright PD, Cheesman E, Giulino-Roth $\mathrm{L}$, et al. Increased risk for malignancies in 131 affected CTLA4 mutation carriers. Front Immunol 2018;9:2012.

21. Impact of NIH Research: National Institutes of Health (NIH) [Internet]. Bethesda (MD): National Institutes of Health; [cited 2018 May 1]. Available from: https://www.nih.gov/about-nih/what-we-do/impact-nihresearch/our-society.

22. Primary immunodeficiencies (2016-2017) [Internet]. Grantome: c2015; [cited 12019 Oct 10]. Available from: http://grantome.com/search?q=pri mary+immunodeficiencies.

23. USIDNET (2016-2017) [Internet]. Grantome: c2015; [cited 2018 Month Day]. Available from: http://grantome.com/search?q=USIDNET.

24. About USIDNET: US Immunodeficiency Network (USIDNET) [Internet]. Towson (MD): USIDNET; [cited 2019 Oct 6]. Available from: https://usidnet.org/.

25. Survey of patients with primary immunodeficiency in Korea [Internet]. Cheongju (Korea): Korea Centers for Disease Control and Prevention (KCDC); [cited 2007 Sep 5]. Available from: http://www.cdc.go.kr/ CDC/notice/CdcKrInfo0201.jsp?menuIds=HOME001-MNU1154$\mathrm{MNU} 0005-\& \mathrm{fid}=28 \& \mathrm{q}_{\text {_type }}=$ title\&q_value $=\% \mathrm{~EB} \% \mathrm{~A} 9 \% \mathrm{~B} 4 \% \mathrm{EC} \%$ 97\%AD\%EA\%B2\%B0\%ED\%95\%8D\&cid=1188\&pageNum=1.

26. Yi ES, Choi YB, Lee NH, Lee JW, Sung KW, Koo HH, et al. Allogeneic hematopoietic cell transplantation in patients with primary immunodeficiencies in Korea: eleven-year experience in a single center. J Clin Immunol 2018;38:757-66.

27. Lee JH, Sohn WY, Park HY, Hwang SJ, Seo WH, Kim SJ, et al. A clinical study of primary immunodeficiency disease in a single center in seoul from 1996 to 2004. Pediatr Allergy Respir Dis 2005;15:368-80.

28. Ju HY, Kang HJ, Hong CR, Lee JW, Kim H, Song SH, et al. Targeted busulfan and fludarabine-based conditioning for bone marrow transplantation in chronic granulomatous disease. Korean J Pediatr 2016; 59:S57-9.

29. Kim JS, Hwang J, Choi YH, Kim WS, Kim JG. A case of esophageal obstruction complicated in a patient with chronic granulomatous disease: esophageal obstruction in chronic granulomatous disease. Korean J Pediatr Infec Dis 2014;21:53-8.

30. Kim L, Kim JA, Kim S. A guide for the utilization of Health Insurance Review and Assessment Service National Patient Samples. Epidemiol Health 2014;36:e2014008.

31. The Sixth Revision of the Korean Standard Classification of Diseases: Statistics Korea [Internet]. Daejeon (Korea): Statistics Korea; [cited 2010 Jul 13]. Available from: http://kostat.go.kr/portal/eng/news/3/index.board ?bmode $=$ read $\&$ aSeq $=71706$. 
32. Classification of Disease (ICD) [Internet]. Geneva (Switzerland): World Health Organization; [cited 2019 Oct 10]. Available from: https://www. who.int/classifications/icd/icdonlineversions/en/.
How to cite this article: Son S, Kang JM, Hahn Y, Ahn K, Kim YJ. Systematic review of literature and analysis of big data from the National Health Insurance System on primary immunodeficiencies in Korea. Clin Exp Pediatr 2021;64:141-8. https://doi.org/10.3345/cep.2019.01347 